\title{
Effect of the fuel-air flow velocity on heat release rate of swirling non-premixed methane flames
}

\author{
Kuanliang Wang ${ }^{\mathrm{a}}$, Fei $\mathrm{Li}^{\mathrm{b}}{ }^{\mathrm{*}}$, Pengfei Zou ${ }^{\mathrm{c}}$, Xin Lin $^{\mathrm{b}}$, Ronghai Mao ${ }^{\mathrm{c}}$, Xilong $\mathrm{Yu}^{\mathrm{b}}$ \\ a School of Aerospace Engineering, Beijing Institute of Technology, Beijing 100081, China \\ b State Key Laboratory of High Temperature Gas Dynamics, Institute of Mechanics, CAS, Beijing 100190, China \\ c Dept. of Discipline Engineering, RED Center, AECC Commercial Aircraft Engine CO., LTD, Shanghai 200241, China
}

\section{A R T I C L E I N F O}

\section{Article history:}

Received 31 January 2019

Received in revised form 19 August 2019

Accepted 7 October 2019

Available online 10 October 2019

\section{Keywords:}

Swirl flame

Heat release

Chemiluminescence

3D-CTC

\begin{abstract}
A B S T R A C T
A laboratory-scale gas turbine model combustor fueled with methane is studied experimentally with the aid of three-dimensional computed tomography of chemiluminescence (3D-CTC) and high speed (5 $\mathrm{kHz}$ ) chemiluminescence imaging. Various fuel-lean operating conditions were tested to investigate the impact of flow velocity on heat release rate oscillation and spatial structure transition with fixed global equivalence ratio of about 0.65 and dump plane velocity of $2.9-18.3 \mathrm{~m} / \mathrm{s}$. In the study of combustion structure transition, the three-dimensional relative emissions of $\mathrm{CH}^{*}$ were measured and taken as qualitative indicators of the heat release rate. This 3D measurement method utilizes $\mathrm{CH}^{*}$ images from 8 directional as inputs combined with tomographic algorithms to compute the 3D distribution of $\mathrm{CH}^{*}$ $\mathrm{CL}$ intensities. For all tested conditions, pronounced extension of inner recirculation zone (IRZ) along the nozzle is observed under the attached ( $V$-shaped) swirl stabilized flames. During the increase of Reynolds number, the heat release zone changing obviously along the nozzle radial and axis direction, and the largest heat release plane moves downstream significantly. In addition, an intensified high-speed camera was adopted for the heat release dynamics study. Strong oscillations appeared in the flame zone that significantly affected the total heat release oscillations, and oscillation increases with the raise of Reynolds number.
\end{abstract}

(c) 2019 Elsevier Masson SAS. All rights reserved.

\section{Introduction}

Swirling burner is vital to gas turbine (GT) combustor, which creates the pressure gradient by the swirl flow and then generate heat reflux to stabilize the combustion. In recent years, increasing attention has been drawn to features of the swirl flames, such as the complex flow-field, thermo-acoustic instability and the flame shape transition. Excellent reviews aspects above may be found elsewhere [1-5] based on the laboratory model combustor by optical diagnosis. Various optical access for laser measurements have been conducted for the swirling $\mathrm{CH}_{4} /$ air diffusion flames at atmospheric pressure with the equivalence ratio between 0.55 and 0.75 by Weigand et al. [1,2]. They measured the flow field by laser Doppler velocimetry, and visualized the flame structures by laserinduced fluorescence (LIF) of $\mathrm{OH}$ and $\mathrm{CH}$ radicals. Concentrations of some major species, temperature, and mixture fraction were determined by laser Raman scattering.

\footnotetext{
* Corresponding author.

E-mail address: lifei@imech.ac.cn (F. Li).
}

In recent years, attention has been drawn to flame shape transitions for the need for understanding and predicting periodic state and transient behaviors in certain combustion systems [3-10]. Renaud et al. [3] studied the flame shape transition in a laboratoryscale swirling burner with the help of high speed $(20 \mathrm{kHz})$ spray PIV and chemiluminescence imaging. In their experiments, flashback happens in the inner recirculation zone (IRZ) in less than $10 \mathrm{~ms}$, and the flame attaches itself to the injector in a tuliplike shape. Cheng et al. [11] found that the flame shape changing was strongly affected by the turbulent flame speed and the reactants burning in the outer recirculation zone (ORZ). The temperature variation as much as $100 \mathrm{~K}$ has been observed on the tip of the buff body during the transitions between attached ( $V$-shaped) and lifted ( $M$-shaped) flames [12]. Nowadays, combustion instability has become one of the most important of issues for the working of gas turbine, and investigations have shown that flame structural change also has effects on the self-excited combustion instabilities [4,13,14]. Lee et al. [13] conducted an experimental study to characterize the instability mechanisms, found that the flame-vortex interaction and the equivalence ratio fluctuation interact each other and determine the instability characteristics in partially premixed conditions. The relation of combustion instabil- 
ity and flame structure indicated using $\mathrm{OH}$ chemiluminescence was examined by Yoon et al. $[4,14]$, and they suggest that pressure fluctuation of combustion causes deformation of flame structure and that variation of flame has a strong effect on combustion instability. During low velocity instability conditions, vortex structures appear at the edge of the flame and it fluctuates at the same frequency as the instability, which radiates the same total heat release rate.

As the identification of combustion reaction zone, the heat release rate is a basis for monitoring the flame state to control combustion oscillation and essential in the study of flame shape transition and combustion dynamic oscillation. The spatial variation of heat release rate has been used for the discusses of the appearance of the reaction zones changing with flame parameters [15]. Stöhr et al. [16] concluded that a significant part of heat release takes place in a tubular structure that is located along the processing vortex core (PVC) and rotates around the axis of swirler. At present, there is no feasible direct measurement method for the heat release rate of swirling flames, most of which use indirect measurement methods based on the emission of chemiluminescence $[3,13,17-19]$, and several studies have shown that $\mathrm{OH}^{*}$ and $\mathrm{CH}^{*}$ are reliable measures for the integral heat releases $[20,21]$. Chemiluminescence can serve as the indicator of heat release rate and then combine with the pressure in the combustor to reveal the combustion instability [13]. Palies et al. [17] assumed that the $\mathrm{OH}^{*}$ emission is proportional to the heat release rate and used the Abel transformation to estimate the distribution of heat-release, the $\mathrm{OH}^{*} \mathrm{CL}$ signal was expected to be a more diffuse signal due to the thermal excitation and transport [22]. Meanwhile, the $\mathrm{CH}^{*}$ has the minimal thermal excitation and its emission falls with the visible wavelength range, which lowers the requirement on imaging system. For the reasons above, the three-dimensional computed tomography of chemiluminescence (3D-CTC) of $\mathrm{CH}^{*}$ is the best solution for obtaining the 3D distribution of heat release rate for swirl flames and the variation of its 3D shape.

The 3D-CTC method was firstly demonstrated by J. Floyd et al. [23,24] for thermal-fluid studies, and is attractive for applications in harsh industrial environments due to the highly desirable of time resolved 3D data. The adoption of fiber-based endoscopes for 3D-CTC experiments, making it much more flexible by not requiring direct line-of-sight configuration [25]. Works on the multiple-projection models were also be made for the reconstruction method [26-28]. In the 3D-CTC application for swirl flames, Mohri et al. [29] illustrates how the locations of the views influence the reconstructions, and discussed the effect of projection numbers on the reconstruction. The evolution of the structural features at $4 \mathrm{kHz}$ have been captured by the CTC method, that suggested a rotating helical structure of the swirl flame [30]. Unfortunately, all those CTC investigations for swirl flames are in the opening environment with no restriction of combustion chamber that makes the dynamic characteristics are far from gas turbine (GT) flame flow fields such as the outer recirculation zone generated due to confinement effects.

The aim of this work is to investigate the effects of flow velocity on heat release rate of swirling non-premixed methane flames both spatially and temporally. Four operating conditions were examined based on a laboratory-scale lean GT-like combustor. The spatial evolution of heat release rate was discussed by the shape transition of $\mathrm{CH}^{*}$ captured by 3D-CTC method. 8 projections with carefully calibrated multi-projection processing parameters were used as inputs of ART (Algebraic Reconstruction Technique) algorithm for tomography computation. The projection model used here is based on the out-of-focus imaging model of large field of view we proposed [31], which takes the intensity distribution over the blurring circle caused by the limited depth of field (DOF) into consideration. In addition, an intensified high-speed camera was

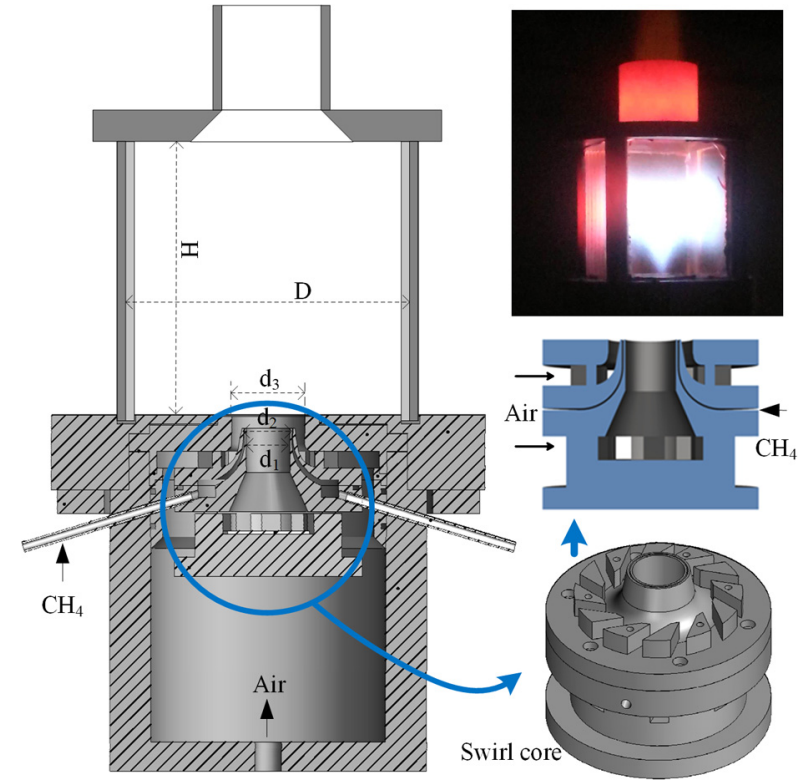

Fig. 1. The model combustor and the 3D-CTC setup.

Table 1

Parameters of the four flames investigated.

\begin{tabular}{|c|c|c|c|c|c|c|c|}
\hline & \multicolumn{2}{|l|}{ Air } & \multicolumn{2}{|l|}{$\mathrm{CH}_{4}$} & \multirow[t]{2}{*}{$\Phi_{\text {glob }}$} & \multirow[t]{2}{*}{$v(\mathrm{~m} / \mathrm{s})$} & \multirow[t]{2}{*}{$\mathrm{Re}$} \\
\hline & $\mathrm{sl} / \mathrm{min}$ & $\mathrm{g} / \mathrm{min}$ & $\mathrm{sl} / \mathrm{min}$ & $\mathrm{g} / \mathrm{min}$ & & & \\
\hline A & 80 & 103.6 & 5.5 & 3.93 & 0.65 & 2.9 & 5099 \\
\hline B & 160 & 207.1 & 10.8 & 7.71 & 0.64 & 5.8 & 10192 \\
\hline $\mathrm{C}$ & 379 & 490.6 & 25.2 & 18.0 & 0.63 & 13.7 & 24129 \\
\hline $\mathrm{D}$ & 505 & 653.8 & 33.8 & 24.1 & 0.64 & 18.3 & 32159 \\
\hline
\end{tabular}

adopted to study the temporally variation characteristics. The oscillations of heat release rate were analyzed based on the behavior of $\mathrm{CH}^{*}$ chemiluminescence under different Reynolds numbers.

\section{Experimental setup and procedures}

\subsection{Gas turbine model combustor and operating conditions}

The atmospheric-pressure gas turbine model combustor used in this work is sketched in Fig. 1, that comprises a section diagram of the whole burner, a state of on fire, a swirl core inside the burner and the schematically shown of gases feeding. The air flows delivered to the flame through the central nozzle and the outermost annular nozzle are co-swirled by the swirl core beforehand. The non-swirling $\mathrm{CH}_{4}$ is fed through the middle annular nozzle from two thin injector tubes with a diameter of $3 \mathrm{~mm}$. The combustion chamber has a square section of $85 \times 85 \mathrm{~mm}$ and a height of $110 \mathrm{~mm}$, and the diameters of three nozzles are $d_{1}=15 \mathrm{~mm}$, $d_{2}=17 \mathrm{~mm}$ and $d_{3}=25 \mathrm{~mm}$, which configuration is designed based on the model combustor of citation [1,2].

Four operating conditions of gas feeding velocity were investigated with the Reynolds numbers from 5000 to 32000, and the global equivalence ratio were all in lean values. The resulting values for volume flow rates, mass flow rates, global equivalence ratio $\left(\Phi_{\text {glob }}\right)$, flow velocities $(v)$ and corresponding Reynolds numbers (Re) are summarized in Table 1 . Air, generated by aircompressor, was pre-dried and then controlled by a mass flow controller (Bronkhorst In-Flow). $\mathrm{CH}_{4}$ was also controlled by a mass flow controller before being divided to two branches and flowed through two thin injector tubes accordingly, and the ignition place of combustor was at the exit of exhaust tube. The operating conditions were chosen because the variation of gas feeding velocity 

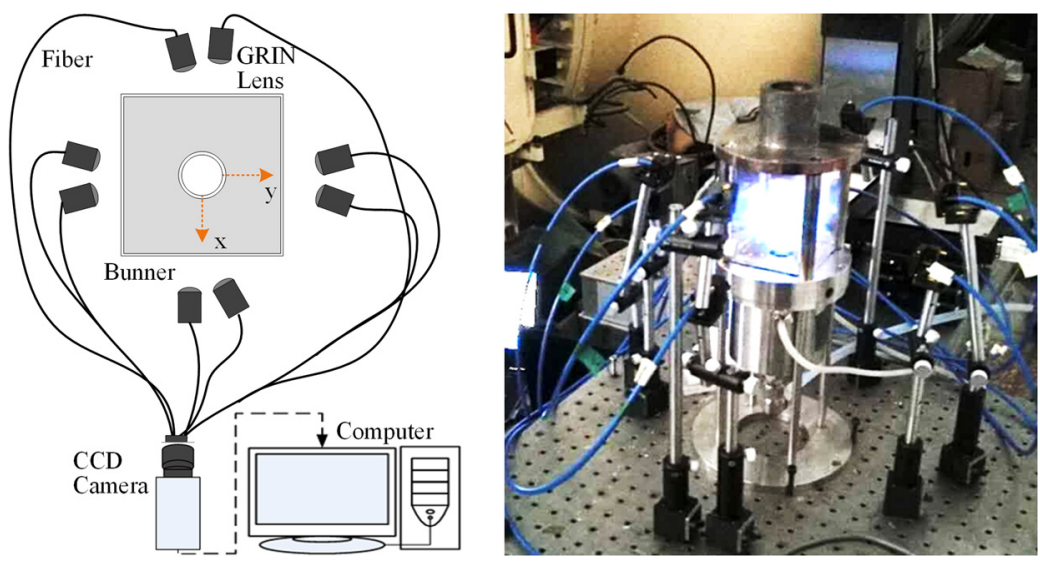

Fig. 2. (a) Multi-directional imaging system [31]; (b) Experimental arrangement of imaging system.
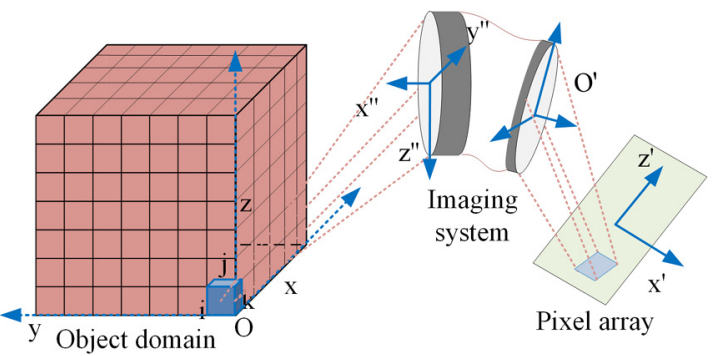

Fig. 3. Physical coordinate system of the 3D-CTC setup.

there showed a strong response of flames and the stability was enough to avoid blow-off.

\subsection{Diagnostic setup and physical formulation}

For the challenging capture of spatial flame mode transition, our multi-directional imaging system used customized $8 \times 1$ endoscopes and only one CCD camera (IMI 147FT). The fiber bundle with eight inputs and one output that can obtain all projection by single shot without synchronized triggering. Gradient-index (GRIN) lens were used before the fiber that establish a field angle of about $50^{\circ}$ [31]. To achieve the best quality of the reconstruction, 8 projections should be scattered as much as possible that we arranged two lenses for each optical window with opposite pitch angles as shown in Fig. 2(a). Narrowband filters (center wavelength at 430 $\mathrm{nm}$, FWHM of $10 \mathrm{~nm}$ ) to block the emission other than $\mathrm{CH}^{*} \mathrm{CL}$. The exposure time was $60 \mathrm{~ms}$ and the 3D-CTC reconstruction was the average intensity during this time period.

The CTC problem is schematically shown in Fig. 3. We use $F$ to denote the distribution of the chemiluminescence intensities in the object domain. The emission intensity recorded by the CCD $(I)$ from a specific viewing angle is then expressed as:

$$
I\left(x^{\prime}, y^{\prime}\right)=\sum_{x_{i}, y_{j}, z_{k}} F\left(x_{i}, y_{j}, z_{k}\right) \cdot W\left(x_{i}, y_{j}, z_{k} ; x^{\prime}, y^{\prime}\right)
$$

where $i, j, k$ are the indices of the voxel; $x^{\prime}, y^{\prime}$ are the indices of the pixels; and $W$ is the weight matrix representing the relationship between a pixel and voxels. For our multi-directional imaging system, the object domain for is a square section of $90 \times 90 \mathrm{~mm}$ with a height of $70 \mathrm{~mm}$ to cover the entire flame, and is discretized into 567,000 voxels $(90 \times 90 \times 70)$, whereas the number of pixels is $627,200(280 \times 280 \times 8)$.

For studying the temporally variation characteristics, a highspeed camera (PHOTRON FASTCAM SA-Z) is vertically to the chamber window with an intensifier (Lambert Instruments) in the front,

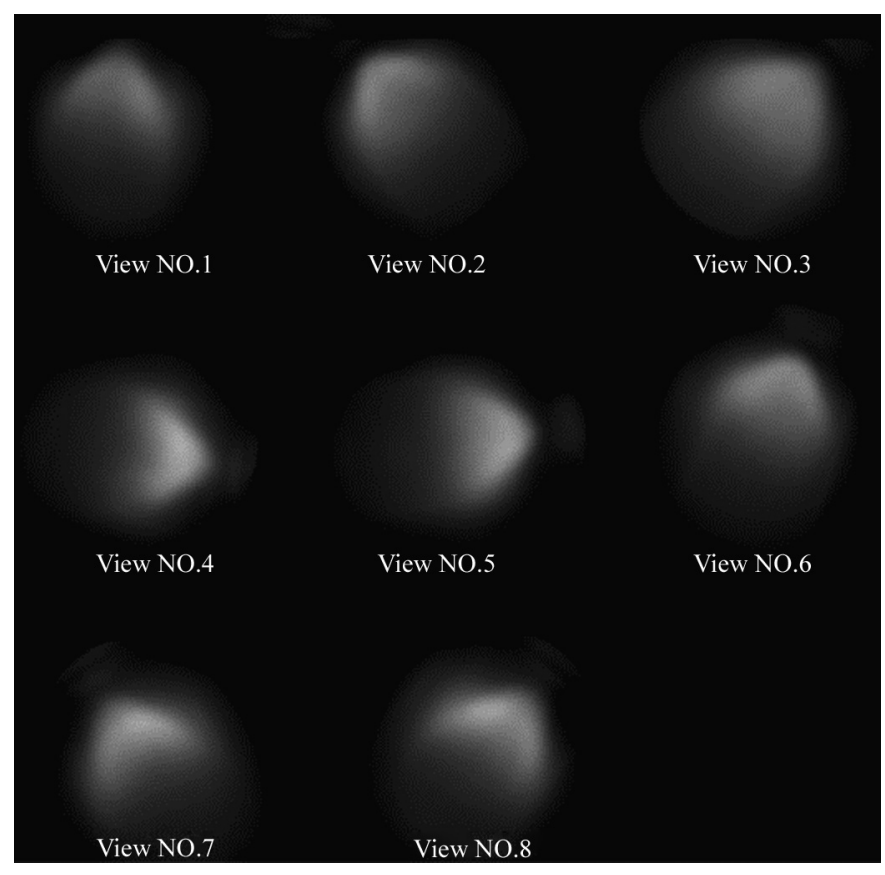

Fig. 4. Simultaneous projections of the flame B.

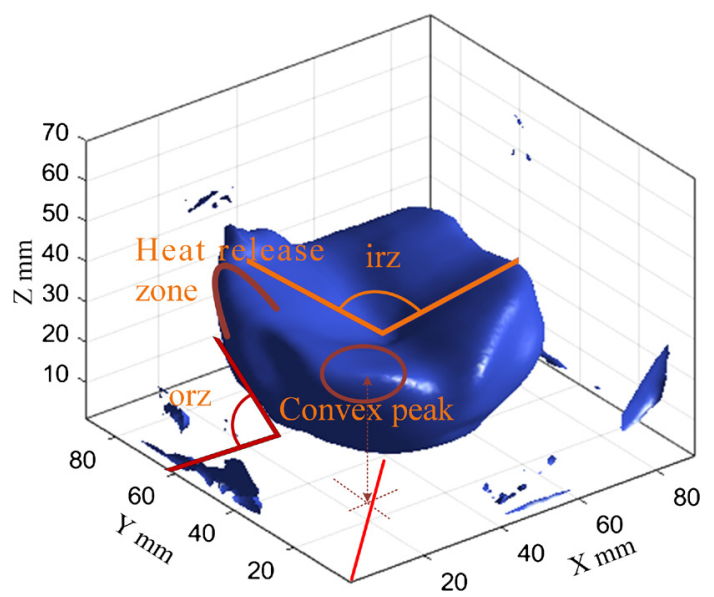

Fig. 5. 3D profile of flame B.

and same optical filter is also used to capture the $\mathrm{CH}^{*}$ chemiluminescence. A commercial lens (Nikon AF $60 \mathrm{~mm} / 2.8 \mathrm{D}$ ) is carefully focused on the center plane of the chamber. The frame rate of the 

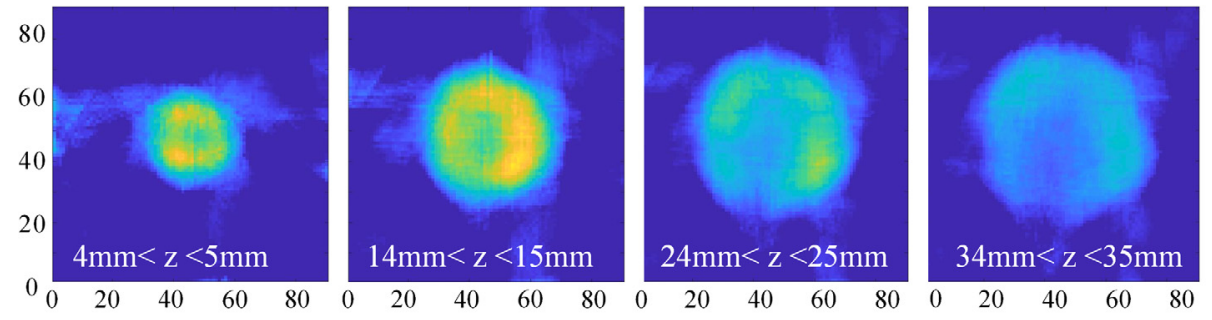

(a)

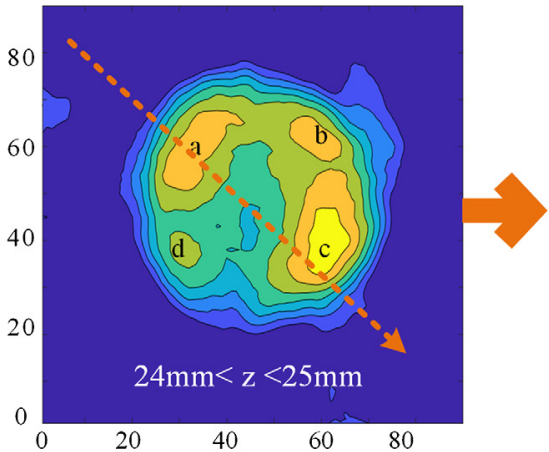

(b)

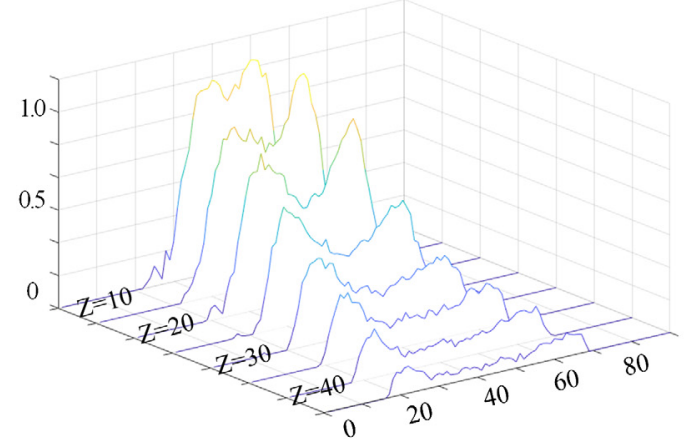

(c)

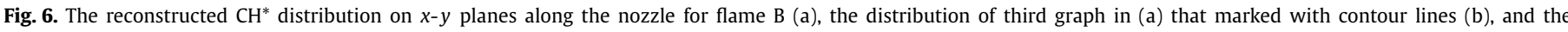
values on diagonal lines for each height (c).

camera is $5 \mathrm{kHz}$ with an exposure of $0.2 \mathrm{~ms}$ for all operating conditions. The resolution of images is $768 \times 808$ pixels corresponding to an $8.89 \times 9.34 \mathrm{~cm}^{2}$ practical interrogation area.

\section{Experimental results and discussion}

\subsection{D-CTC reconstruction}

After the arrangement of imaging system, the weight matrix was calculated by the intrinsic parameters which were calibrated based on lens imaging theory. The calibration results show that the distances between lens and the coordinate center of the object domain $\left(90 \times 90 \times 70 \mathrm{~mm}^{3}\right)$ are from $135 \mathrm{~mm}$ to $170 \mathrm{~mm}$, meaning a largest viewing angle of about $37^{\circ}$. The projections of $\mathrm{CH}^{*}$ chemiluminescence were then obtained, as shown in Fig. 4 (flame B), and were inputted to the reconstruction algorithm to compute the distributions of heat release rate. Fig. 5 illustrates the 3D profiles of heat release rate for the responding flames (just flame B was showed here), and the reconstructed shapes for all flames are not much different from each other.

A $V$-shape liked zone can be seen clearly from flame $B$, that an inner recirculation zone and an outer recirculation zone are formed by the strong swirling effects and confinement. Between the IRZ and ORZ, the main heat release zone interacts strongly with the swirling flow field. The 3D structure of the main heat release zone is not axisymmetric to the nozzle axis, as shown in Fig. 5, with four convex peaks closed to the corners of square section that means the heat accumulation is more likely to appears in the chamber corners than the near front-wall. This may be due to the fact that the main non-axisymmetric factor is the square section layout of chamber which caused the restriction of flow field. To reveal the spatially resolved information of the heat release, the reconstructed 2D distribution of $\mathrm{CH}^{*} \mathrm{CL}$ at five $x-y$ planes are shown in Fig. 6(a) with an $z$-direction interval of $10 \mathrm{~mm}$. The outline of the distributions on each plane is an irregular circle, that the diameter increases along the $z$-axis while the heat release values increase rapidly at first and then decrease slowly. A distinct low-heat release zone appears in core of flame corresponding to the IRZ in Fig. 5 and disappears gradually with the height in-

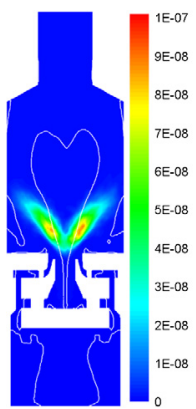

(a)

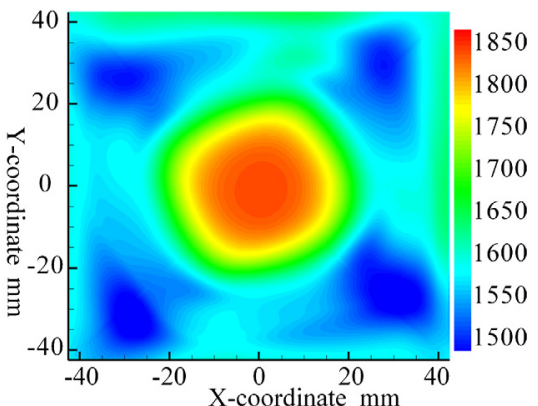

(b)
Fig. 7. Predicted contours of the $\mathrm{CH}$ mole fraction (a) and temperature (b). (For interpretation of the colors in the figure(s), the reader is referred to the web version of this article.)

creases. Fig. 6(b) shows the detailed distinction of the third plane in Fig. 6(a) that marked by contour lines. Four peak values reveled the inner non-axisymmetric heat release rate and lead to the outline of Fig. 5. It indicates that Abel deconvolution could not be used for 3D analysis for the swirl burner used here with square cross-section. Also, the changing along the diagonal lines for each height were shown in Fig. 6(c), which illustrates a more regular processes with spatial location.

Similar changing behavior of temperature is also observed by the numerical simulations by the aid of ANSYS FLUENT with the large eddy simulation (LES). Detailed reaction kinetics of GRI 3.0 was applied and the flamelet Generated Manifold (FGM) method is used to reduce combustion chemistry. As shown in the $\mathrm{CH}$ and temperature profiles in Fig. 7. The model of the swirl injector was of the same structure and size of that used in the experiment, and the contours were obtained by the time averaged data. The contours of $\mathrm{CH}$ concentration in Fig. $7(\mathrm{a})$ is in $x-z$ cross section of nozzle center $(y=45 \mathrm{~mm})$, and the distribution of temperature in Fig. 7(b) is calculated at the height of $40 \mathrm{~mm}$. It should be noticed that the calculated $\mathrm{CH}$ concentration is the low-sate $\mathrm{CH}$ but not the excited $\mathrm{CH}$ species $\left(\mathrm{CH}^{*}\right)$, which is difficult to simulate due to the deficiency of exact formation mechanism. Even though, $\mathrm{CH}$ 


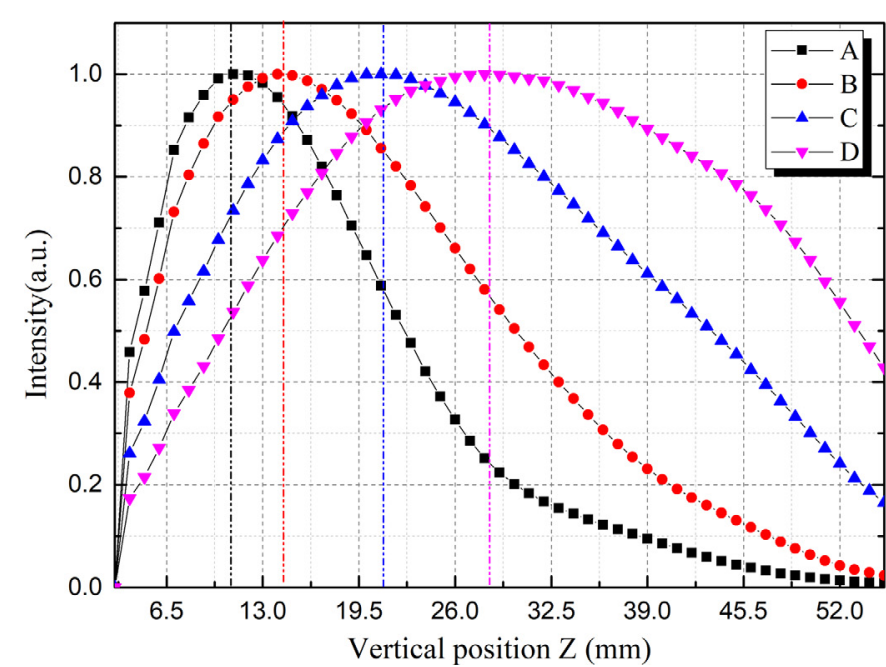

(a)

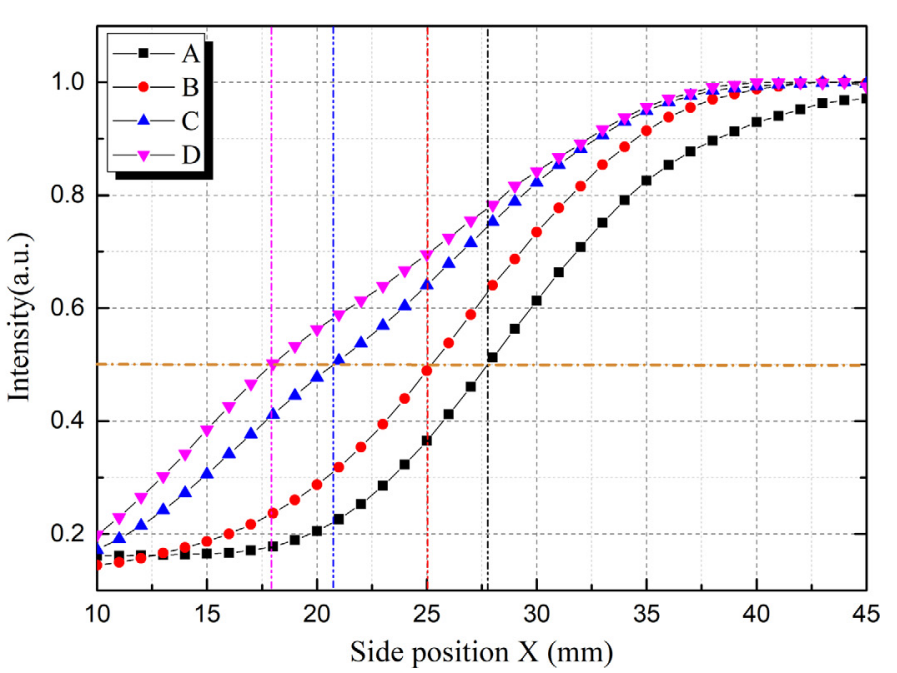

(b)

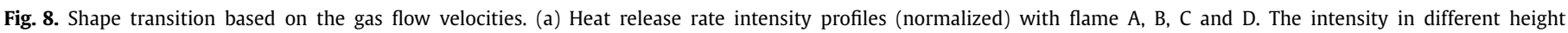

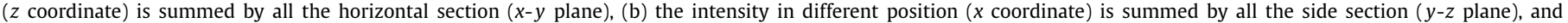
the axis of nozzle is at the coordinate of $45 \mathrm{~mm}$.

concentration can be used to indicate the reaction zone and its distribution shows the $V$-shaped flame structure. High temperature zone suggests the heat release region, its size can be used to compare with the reconstructed 3D profiles as shown in Fig. 5. The reconstructed flame structure and its size agree well with the CFD simulation results. Furthermore, there are four low temperature zones at the corner of square section, which means the restrict effect of boundaries is truly nonuniform on the heat release region.

In order to facilitate the quantitative analysis of the heat release characteristics of the swirling flames, the horizontal and vertical intensity changing under four conditions were compared. As shown in Fig. 8, the curves of distribution (a) is along the nozzle ( $Z$ axis in Fig. 5), and is the sum of voxels on each $x-y$ plane that can reveal the overall heat release characteristics axially. We can see the maximum heat release appears at the height of $10.8 \mathrm{~mm}$, $14.5 \mathrm{~mm}, 21.1 \mathrm{~mm}$ and $28.3 \mathrm{~mm}$ for flame A, B, C and D accordingly, that the main heat release region moves downstream with the increase in Reynolds number. The distribution (b) is along the direction vertical to the nozzle, and is the sum of voxels on each $y-z$ plane that can reveal the overall heat release characteristics in the horizontal direction. The curves in (b) just shows the half part of flames from the near wall point $(x=10 \mathrm{~mm})$ to the axis of nozzle $(x=45 \mathrm{~mm})$ and the rest half is approximately axisymmetric to it. The profiles of four flames show that the half of full-width at half-maximum for major heat release is about $17.3 \mathrm{~mm}, 20.1 \mathrm{~mm}$, $24.3 \mathrm{~mm}$ and $27.0 \mathrm{~mm}$ for flame A, B, C and D.

The size of heat release zone increases with the increase of vertical and horizontal position. In order to analyze the opening angle of IRZ, the centroids of heat release zone on the section across nozzle center $(y=45 \mathrm{~mm}$ ) were calculated. As shown in Fig. 9, the abscissa is the centroid coordinates of $Z$ and the ordinate is the distance from centroid to the axis of nozzle. It is clear that, centroid coordinates become far away from the axis of nozzle when the height increases, which is in agreement with the $V$ shape exhibited in Fig. 5. The increments of centroid position are similar for all flames that means the contours of the IRZ are nearly identical for all four flames, agreeing with the results of Weigand et al. [1]. The mean heat release position is not subject to the change of flow velocity although the region can be enlarged at higher flow rate. In order to reveal the spatial resolved relationship between the flow velocity and the heat release, the local net heat release rate based on first law of thermodynamics was applied [32] as:

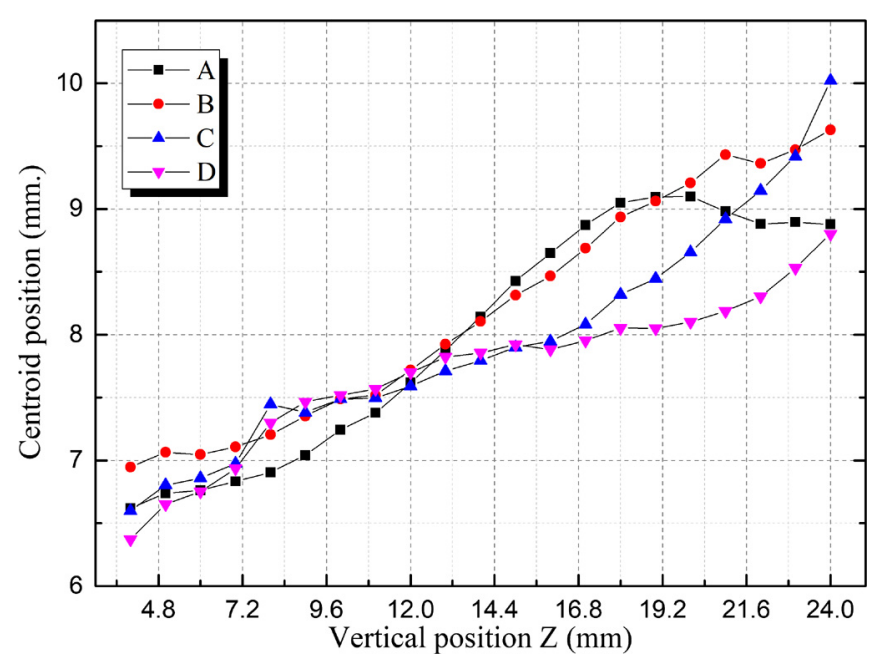

Fig. 9. Centroids of the flames on the section of $y=45 \mathrm{~mm}$ representing the extension of IRZ for flames.

$\dot{Q}_{n e t}=\rho(\phi, \gamma) \cdot c_{p}(\phi, \gamma) \cdot(\vec{v} \circ \nabla T)$

where $\dot{Q}_{n e t}$ is the heat release rate of flame volumetric net, $\rho$ is the fluid density, $c_{p}$ is the isobaric heat capacity, $\vec{v}$ is the fluid velocity, and $\nabla T$ is the temperature gradient. The formulation implies that the increase of the sensible enthalpy of the fluid was caused by the heat released from the combustion. $\rho$ and $c_{p}$ are the functions of the fluid composition that can be specified by the local equivalence ratio $\phi$ and the time averaged progress variable of combustion $\gamma$.

For the four cases in current study, the local equivalence ratios are almost the same. Based on the results in Figs. 8 and 9, similar heat release distributions for these cases indicate that the variance of $\gamma$ and temperature gradient should not be unremarkable compared with the velocity vector. Increasing flow rate means higher heat-release rate along the edge of the $V$-shape and the flame moves forward due to the flame speed. Therefore, maximum heat release rate intensity moves along the axis of nozzle as shown in Fig. 8(a), and the heat release region enlarged correspondingly in Fig. 8(b). As a contrast, the direction of velocity changes little as well as the centroid position of the heat release as shown in Fig. 9. 


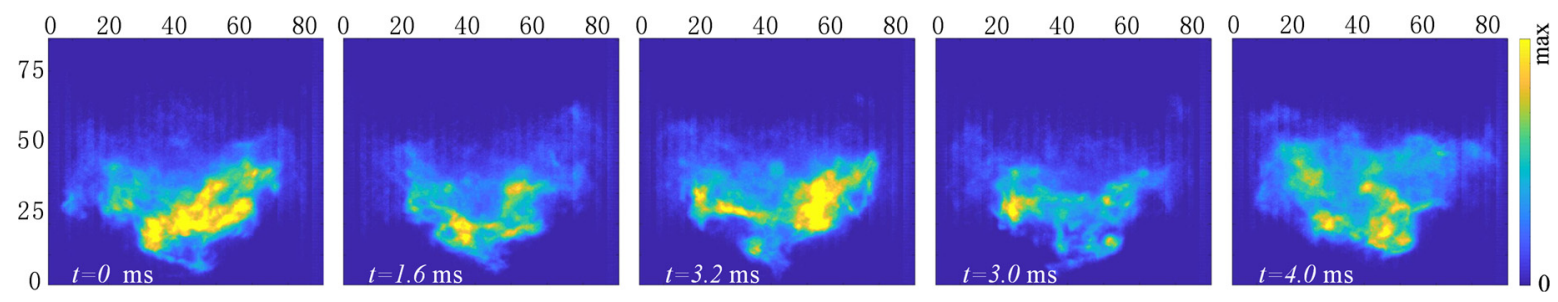

Fig. 10. Typical time sequence of the integrated heat release rate for flame D.

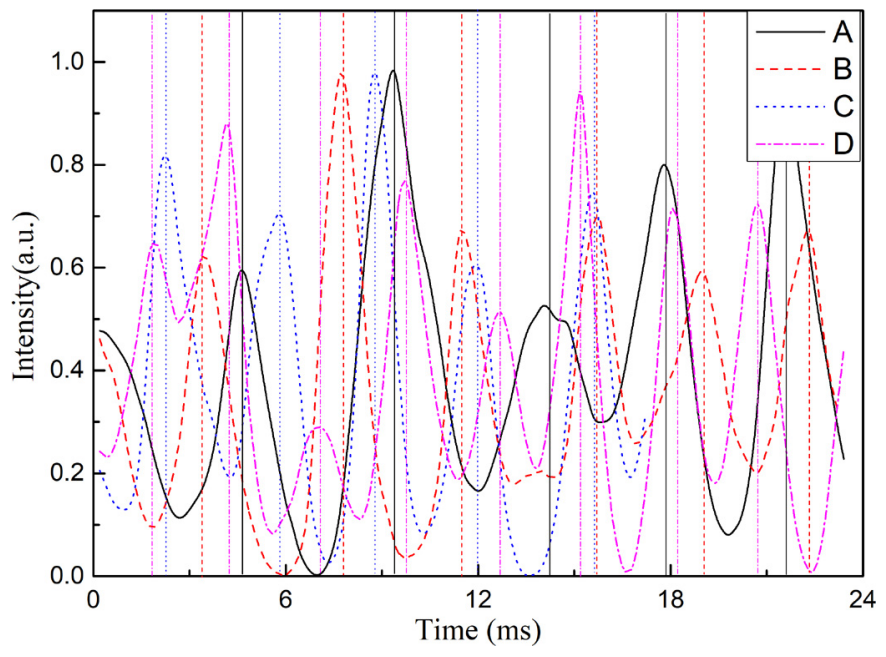

Fig. 11. Temporal history of the heat release rate for flame A, B, C and D.

\subsection{Dynamics analysis}

Analyses [33,34] have been provided that the total chemiluminescence signal, integrated over the combustor volume, fluctuated at exactly the thermo-acoustic frequency. Fig. 10 shows the temporal evolution of the chemiluminescence field (flame D) over a short interval, during which the signal moved periodically. As can be seen, the time of most heat release appearing on right side is set to zero, and then the location moves around the burner. When the most heat release appears again on right side, the time span of the motion is $3.2 \mathrm{~ms}$. Fig. 11 shows temporal histories of the total chemiluminescence signal for all flames. The intensity peaks are separated by different period of time, and these variations on oscillation period indicate a changing of thermo-acoustic frequency. The averaged separation for flame A, B, C and D is about $4.2 \mathrm{~ms}$, $3.8 \mathrm{~ms}, 3.3 \mathrm{~ms}$ and $2.7 \mathrm{~ms}$, corresponding to a thermo-acoustic frequency of $238 \mathrm{~Hz}, 263 \mathrm{~Hz}, 303 \mathrm{~Hz}, 370 \mathrm{~Hz}$.

The frequency variations of thermo-acoustic shows that the fluctuation of heat release rate was greatly affected by the flow velocity, that is, as the gas feeding velocity raising, in one hand, it caused the motion of PVC around the burner enhanced, and then the periodic asymmetry in the flame surface increased accordingly, on the other, the confinement effects on inflow became more obvious and the oscillations of flow fields were intensified.

\section{Conclusion}

For studying the effect of the flow velocity on heat release rate of swirling flame in a laboratory-scale lean-fuel GT-like combustor, a fiber-based 3D-CTC system was used to capture the 3D shape of the flame emission $\left(\mathrm{CH}^{*}\right)$ and thus the 3D heat release rate is identified. Based on the 3D imaging, a $V$-shape reaction zone can be clearly seen, indicating the IRZ and ORZ are formed by the strong swirling effects and confinement. Both the 3D outline shape and the inner distribution shows a non-axisymmetric structure along the nozzle, and four obvious peak values exist near the corner of square section due to the restriction of flow field by the square section shape chamber. Comparing with cases at different flow rates, it can be found the main heat release region moves downstream with the increase in flow velocity while the contours of the IRZ are nearly identical for all cases. High speed 2D imaging shows thermo-acoustic frequency of $238-370 \mathrm{~Hz}$ for the four cases and it increased when the Reynolds number increased indicating a strong flow-flame interaction of swirl flames.

\section{Declaration of competing interest}

There is no competing interest.

\section{Acknowledgements}

This work was sponsored by the National Natural Science Foundation of China (Grant 11372329) and supported by the Scientific Instrument Developing Project of the Chinese Academy of Sciences (Grant YZ201637).

\section{References}

[1] P. Weigand, W. Meier, X.R. Duan, W. Stricker, M. Aigner, Investigations of swir flames in a gas turbine model combustor: I. Flow field, structures, temperature, and species distributions, Combust. Flame 144 (2006) 205-224.

[2] W. Meier, X.R. Duan, P. Weigand, Investigations of swirl flames in a gas turbine model combustor - II. Turbulence-chemistry interactions, Combust. Flame 144 (2006) 225-236.

[3] A. Renaud, S. Ducruix, P. Scouflaire, L. Zimmer, Flame shape transition in a swirl stabilised liquid fueled burner, Proc. Combust. Inst. 35 (2015) 3365-3372.

[4] J. Yoon, M.-K. Kim, J. Hwang, J. Lee, Y. Yoon, Effect of fuel-air mixture velocity on combustion instability of a model gas turbine combustor, Appl. Therm. Eng. 54 (2013) 92-101.

[5] U.E. Meier, D. Wolff-Gaßmann, W. Stricker, LIF imaging and 2D temperature mapping in a model combustor at elevated pressure, Aerosp. Sci. Technol. 4 (2000) 403-414.

[6] L.X. Zhou, Comparison of studies on flow and flame structures in different swirl combustors, Aerosp. Sci. Technol. 80 (2018) 29-37, https://doi.org/10.1016/j.ast. 2018.06.032.

[7] A. Andreini, T. Bacci, M. Insinna, et al., Modelling strategies for the prediction of hot streak generation in lean burn aeroengine combustors, Aerosp. Sci. Technol. (2018) 266-277.

[8] S. Chen, D. Zhao, Numerical study of non-reacting flowfields of a swirling trapped vortex ramjet combustor, Aerosp. Sci. Technol. (2018) 81-92.

[9] Y. Tian, X. Zeng, S. Yang, et al., Experimental study on the effect of equivalence ratio and injector position on flow structure and flame development in the scramjet combustor, Aerosp. Sci. Technol. (2018) 9-19.

[10] R. Feng, J. Li, Y. Wu, et al., Experimental investigation on gliding arc discharge plasma ignition and flame stabilization in scramjet combustor, Aerosp. Sci. Technol. (2018) 145-153.

[11] R.K. Cheng, D. Littlejohn, P.A. Strakey, T. Sidwell, Laboratory investigations of a low-swirl injector with $\mathrm{H}_{2}$ and $\mathrm{CH}_{4}$ at gas turbine conditions, Proc. Combust. Inst. 32 (2008) 3001-3009.

[12] Z. Yin, P. Nau, W. Meier, Responses of combustor surface temperature to flame shape transitions in a turbulent bi-stable swirl flame, Exp. Therm. Fluid Sci. 82 (2017) 50-57.

[13] H.J. Lee, K.T. Kim, J.G. Lee, B.D. Quay, D.A. Santavicca, An Experimental Study on the Coupling of Combustion Instability Mechanisms in a Lean Premixed Gas Turbine Combustor, 2009.

[14] J. Yoon, M.-C. Lee, S. Joo, J. Kim, Y. Yoon, Instability mode and flame structure analysis of various fuel compositions in a model gas turbine combustor, J. Mech. Sci. Technol. 29 (2015) 899-907. 
[15] U. Stopper, M. Aigner, W. Meier, R. Sadanandan, M. Stohr, I.S. Kim, Flow field and combustion characterization of premixed gas turbine flames by planar laser techniques, J. Eng. Gas Turbines Power 131 (2009).

[16] M. Stohr, R. Sadanandan, W. Meier, Phase-resolved characterization of vortexflame interaction in a turbulent swirl flame, Exp. Fluids 51 (2011) 1153-1167.

[17] P. Palies, D. Durox, T. Schuller, S. Candel, The combined dynamics of swirler and turbulent premixed swirling flames, Combust. Flame 157 (2010) 1698-1717.

[18] Z. Wang, F. Li, H. Gu, X.L. Yu, X. Zhang, Experimental study on the effect of combustor configuration on the performance of dual-mode combustor, Aerosp. Sci. Technol. 42 (2015) 169-175.

[19] R. Feng, J. Li, Y. Wu, J.J. Zhu, X.L. Song, X.P. Li, Experimental investigation on gliding arc discharge plasma ignition and flame stabilization in scramjet combustor, Aerosp. Sci. Technol. 79 (2018) 145-153.

[20] T.P. Clark, D.A. Bittker, A Study of the Radiation From Laminar and Turbulent Open Propane-Air Flames as a Function of Flame Area, Equivalence Ratio, and Fuel Flow Rate, Technical Report Archive \& Image Library, 1954, 34(12).

[21] Ludwig C. Haber, Uri Vandsburger, William R. Saunders, et al., An examination of the relationship between chemiluminescent light emissions and heat release rate under non-adiabatic conditions, in: Asme Turbo Expo: Power for Land, Sea, \& Air, American Society of Mechanical Engineers, 2000.

[22] J.S. Hardi, W. Zach Hallum, C. Huang, et al., Approaches for comparing numerical simulation of combustion instability and flame imaging, J. Propuls. Power (2015) 1-16.

[23] J. Floyd, P. Geipel, A.M. Kempf, Computed tomography of chemiluminescence (CTC): instantaneous 3D measurements and Phantom studies of a turbulent opposed jet flame, Combust. Flame 158 (2011) 376-391.

[24] J. Floyd, A.M. Kempf, Computed tomography of chemiluminescence (CTC): high resolution and instantaneous 3-D measurements of a Matrix burner, Proc. Combust. Inst. 33 (2011) 751-758.
[25] M. Kang, Y. Wu, L. Ma, Fiber-based endoscopes for 3D combustion measurements: view registration and spatial resolution, Combust. Flame 161 (2014) 3063-3072.

[26] W. Cai, X. Li, F. Li, L. Ma, Numerical and experimental validation of a threedimensional combustion diagnostic based on tomographic chemiluminescence, Opt. Express 21 (2013) 7050-7064.

[27] J. Wang, Y. Song, Z.H. Li, A. Kempf, A.Z. He, Multi-directional 3D flame chemiluminescence tomography based on lens imaging, Opt. Lett. 40 (2015) 1231-1234.

[28] W. Cai, X. Li, Y. Cao, J. Wang, L. Ma, Practical Aspects of Three-Dimensional Flame Imaging Using Tomographic Chemiluminescence, 2014.

[29] K. Mohri, S. Görs, J. Schöler, A. Rittler, T. Dreier, C. Schulz, A. Kempf, Instantaneous 3D imaging of highly turbulent flames using computed tomography of chemiluminescence, Appl. Opt. 56 (2017) 7385-7395.

[30] T. Yu, C. Ruan, H. Liu, W. Cai, X. Lu, Time-resolved measurements of a swirl flame at $4 \mathrm{kHz}$ via computed tomography of chemiluminescence, Appl. Opt. 57 (2018) 5962-5969.

[31] K. Wang, F. Li, H. Zeng, X. Yu, Three-dimensional flame measurements with large field angle, Opt. Express 25 (2017) 21008.

[32] M. Lauer, M. Zellhuber, T. Sattelmayer, et al., Determination of the heat release distribution in turbulent flames by a model based correction of $\mathrm{OH}^{*}$ chemiluminescence, J. Eng. Gas Turbines Power 133 (12) (2011) 121501.

[33] A.M. Steinberg, I. Boxx, M. Stohr, C.D. Carter, W. Meier, Flow-flame interactions causing acoustically coupled heat release fluctuations in a thermo-acoustically unstable gas turbine model combustor, Combust. Flame 157 (2010) 2250-2266.

[34] I. Boxx, M. Stohr, C. Carter, W. Meier, Temporally resolved planar measurements of transient phenomena in a partially pre-mixed swirl flame in a gas turbine model combustor, Combust. Flame 157 (2010) 1510-1525. 